\title{
Three years of ULTRASPEC at the Thai 2.4-m telescope: Capabilities and scientific highlights
}

\author{
Ram Kesh Yadav $^{1 *}$, Andrea Richichi ${ }^{1,2}$, Puji Irawati ${ }^{1}$, Vikram Singh Dhillon ${ }^{3}$, \\ Thomas R. Marsh ${ }^{4}$, Boonrucksar Soonthornthum ${ }^{1}$
}

${ }^{1}$ National Astronomical Research Institute of Thailand 260 Moo 4, Donkaew, Maerim, Chiangmai,

\author{
50180 Thailand \\ ${ }^{2}$ INAF - Osservatorio Astrofisico di Arcetri, Largo E. Fermi 5, 50125 Firenze, Italy \\ ${ }^{3}$ Department of Physics and Astronomy, University of Sheffield, Sheffield S3 7RH, UK \\ ${ }^{4}$ Department of Physics, University of Warwick, Coventry CV4 7AL, UK
}

\begin{abstract}
High temporal resolution observations enable the study of rapid phenomena such as the flux variations in binary system objects, e.g. cataclysmic variables, compact binary systems, the flux variations in young star clusters, stellar occultations and more. The 2.4-m Thai National Telescope (TNT) is ideally suited for this niche research, being the largest facility in Southeast Asia and being equipped with ULTRASPEC, a high-speed imager based on a low-noise frame transfer electron-multiplying CCD. In the sub-window mode, ULTRASPEC can record uninterrupted sequences with frame rates as fast as few milliseconds. We present some of the key results obtained in the area of high time resolution with ULTRASPEC. We also present the results of a recent worldwide campaign to observe the current series of lunar occultations of Aldebaran ( $\alpha$ Tauri) carried out in close collaboration with the Devasthal facilities, the out-of-eclipse variations on the post common-envelope system J1021+1744, and pre-main-sequence variables in young open cluster Stock 8 .
\end{abstract}

\section{Introduction}

High time resolution optical photometry can be defined as photometry obtained on the time-scales of tens of seconds and below. This is a technological definition, based on the fact that read-out time for the conventional CCDs mounted on the vast majority of the world's largest telescopes is found to be tens of seconds or longer (Dhillon et al. 2014). High time resolution time series photometry is vital to study compact stellar remnants, such as white dwarfs, neutron stars, and black holes. This is due to the fact that the dynamical time-scales for such objects range from seconds to milliseconds and hence associated physical parameters (e.g. structure, radii) and the emission mechanism (e.g. Littlefair et al. 2006) can only be studied by making high-speed observations.

High-speed photometry can also be useful to study the objects having dynamical time-scales of a minute. For example, high time resolution observations of eclipses, transits and occultations of exoplanets, brown dwarfs and Solar system objects can provide excellent spatial resolution and can

*ram_kesh@ @arit.or.th 
be used to search for small variations in their orbits due to presence of other bodies (e.g. Zalucha et al. 2007, Ortiz et al. 2012, Marsh et al. 2014, Richichi et al. 2014). As some of these objects can be very bright (e.g. host stars of exoplanets), a short exposure with negligible dead time is beneficial to avoid saturation without destroying the efficiency of the observations (Dhillon et al. 2014).

In view of all that has been mentioned so far, one may think that high-speed photometry is limited to objects with a dynamical time-scale of few milliseconds to few minutes. However, high cadence photometry can also be beneficial to investigate small-scale variations (due to flare, circumstellar material, oscillations) in long period variables such as main-sequence (MS) and pre-main-sequence (PMS) stars in clusters.

\section{ULTRASPEC at the 2.4-m TNT}

ULTRASPEC is a modified version of ULTRACAM which is an ultra-fast, triple-beam CCD camera designed to study high temporal astrophysical phenomena (Dhillon et al. 2007b). It was originally designed as a bare electron-multiplying CCD (EMCCD) to be used as a spectrograph with the aim to understand the kinematics of compact objects. ULTRASPEC was tested on the sky for the first time in December 2006 on the ESO Faint Object Spectrograph and camera Version 2 (EFOSC2) (Dhillon et al. 2007a). Afterwards, as a visiting instrument, ULTRASPEC was used for two science runs (17 nights in January 2008 and 22 nights in June 2009) with EFOCS2 mounted on the ESO 3.6-m and 3.5-m New Technology Telescope (NTT) at La Silla (Dhillon et al. 2014).

Being a visiting instrument, the duration of the observations with ULTRASPEC was limited to a few weeks due to the time allocation process. Hence, it was difficult to carry out long-term and dedicated monitoring of new astronomical discoveries (Dhillon et al. 2014). Therefore, in order to find a permanent home for ULTRASPEC on the 2.4-m Thai National Telescope (TNT ${ }^{1}$ ), in 2011, Dhillon and his team signed a Memorandum of Understanding with the National Astronomical Research Institute of Thailand (NARIT). ULTRASPEC was installed on TNT in August 2013 and saw first light in November 2013.

ULTRASPEC is a $1024 \times 1024$-pixel frame-transfer EMCCD detector with a pixel scale of $0.45^{\prime \prime} /$ pixel giving a field of view (FoV) of $7.7^{\prime} \times 7.7^{\prime}$. Such a FoV provides a $80 \%$ probability to find a comparison star of magnitude $R=11$ at a galactic latitude of $30^{\circ}$ as per the star count listed by Simons (1995). Brighter comparison stars ensure reliable differential photometry. A number of filters are available with ULTRASPEC which includes Sloan Digital Sky Survey (SDSS) and H $\alpha$ filters (See Dhillon et al. 2014; Table 2). Fig. 1 (left) shows the mechanical drawing of ULTRASPEC and Fig. 1 (right) is a picture of ULTRASPEC when mounted on the Nasmyth focus of the TNT.

ULTRASPEC uses an e2v CCD 201-20 as its detector having a pixel size of $13 \mu \mathrm{m}$. The chip is thinned, back-illuminated, and coated with e2v's standard midband anti-reflection coating, providing a maximum quantum efficiency (QE) of approximately $96 \%$ around $600 \mathrm{~nm}$ (see Fig. 3 of Dhillon et al. 2014). The full well capacity of each pixel is $\sim 80000 \mathrm{e}^{-}$and the CCD is operated with a system gain of $g_{s} \sim 0.8 \mathrm{e}^{-} / \mathrm{ADU}$. The CCD has two switchable outputs (normal and avalanche), as described in Fig. 5 of Dhillon et al. (2014). Depending upon the observers' requirements, ULTRASPEC can be read out in a number of different modes: full-frame mode, one-window, two-windows, threewindows, four-windows and drift mode. The frame rate obtained in each mode can be adjusted by modifying the positions and/or sizes of the windows on the CCD, the binning factor, and the read-out speed. It should be noted that while selecting multiple windows, they must not overlap in vertical direction.

\footnotetext{
${ }^{1}$ More information about TNT can be found at http://www.narit.or.th/en/index.php/observing-facilities/thai-nationalobservatory-tno/2-4-m-telescope/specification
} 

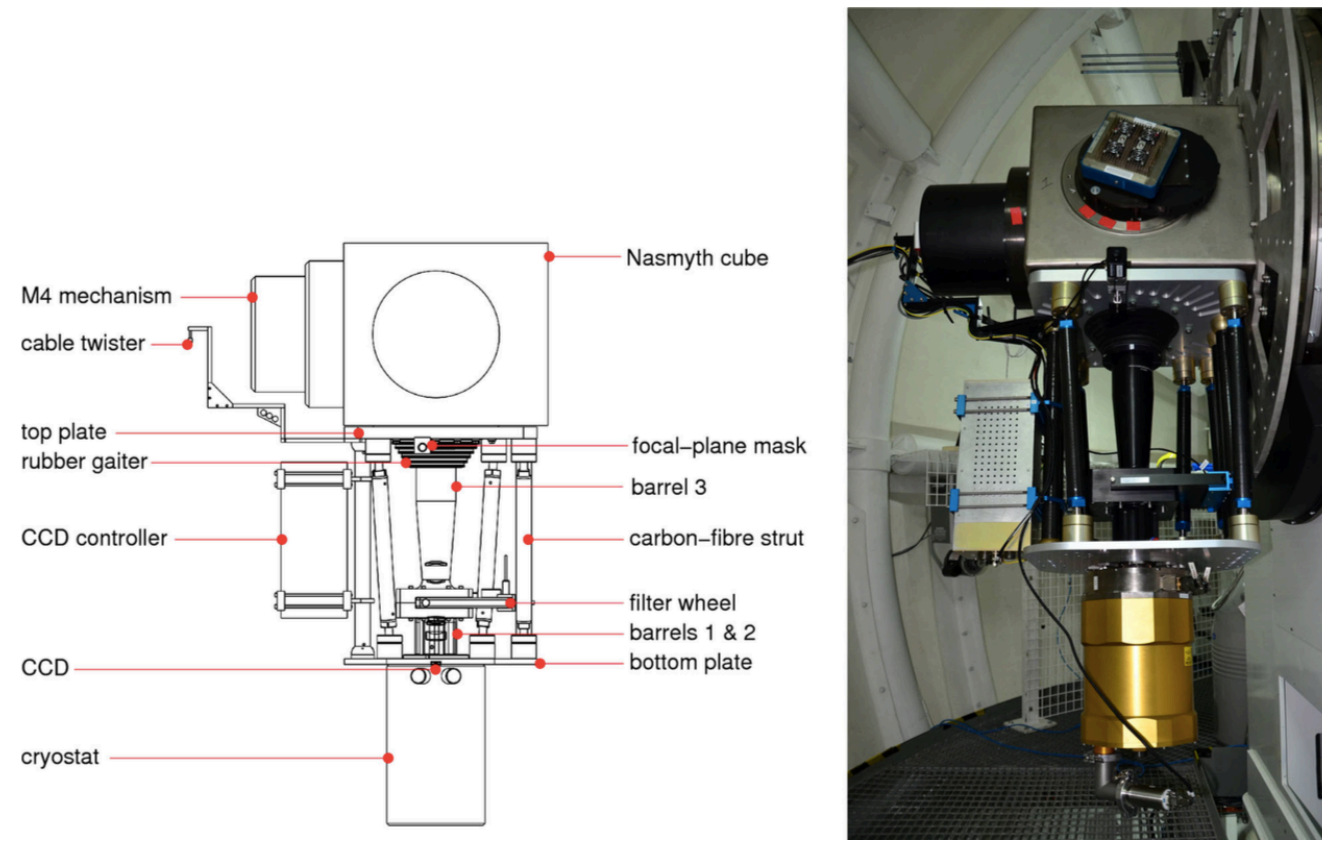

Figure 1: Left: Mechanical drawing of the ULTRASPEC optomechanical chassis, highlighting some of the components shown in the picture on the right. Right: Picture of ULTRASPEC mounted on the Nasmyth focus of the TNT (from Dhillon et al. 2014).

A detailed description and recent updates about ULTRASPEC at 2.4-m TNT can be found in Dhillon et al. (2014) and on the instrument's webpage ${ }^{2}$. In the next section we summarize a few recent results based on ULTRASPEC observations.

\section{Recent Results}

\subsection{Lunar Occultation: Asymmetries in the Aldebaran Photosphere}

Aldebaran ( $\alpha$ Tau), a K5 giant, is one of the brightest and most distinctive stars in the sky. As a result, it has one of the longest records of observations and publications. Located just at $20 \mathrm{pc}$ from the Sun, it has one of the largest diameters among all stars. Hence it has been subject to several measurements using a number of techniques. Richichi et al. (2017) reported photospheric asymmetries of $\alpha$ Tau using the lunar occultation (LO) technique. They recorded three LO light curves of $\alpha$ Tau using the Devasthal 1.3-m telescope in India, 2.4-m TNT in Thailand and SAO 6-m telescope in Russia (see Table 1 of Richichi et al. 2017). Fig. 2 shows light curves obtained from these three telescopes.

The recorded light curves have been analyzed using both model dependent and model independent methods. The conventional uniform-disk and limb-darkened disk diameter were estimated to be 20.3 milliarcseconds on average which are approximately consistent with a diameter estimated using longbaseline interferometry. Richichi et al. (2017) have also suggested a surface asymmetry which could be due to the presence of cool spots.

\footnotetext{
${ }^{2}$ http://www.vikdhillon.staff.shef.ac.uk/ultraspec/ultraspec_tnt.html
} 


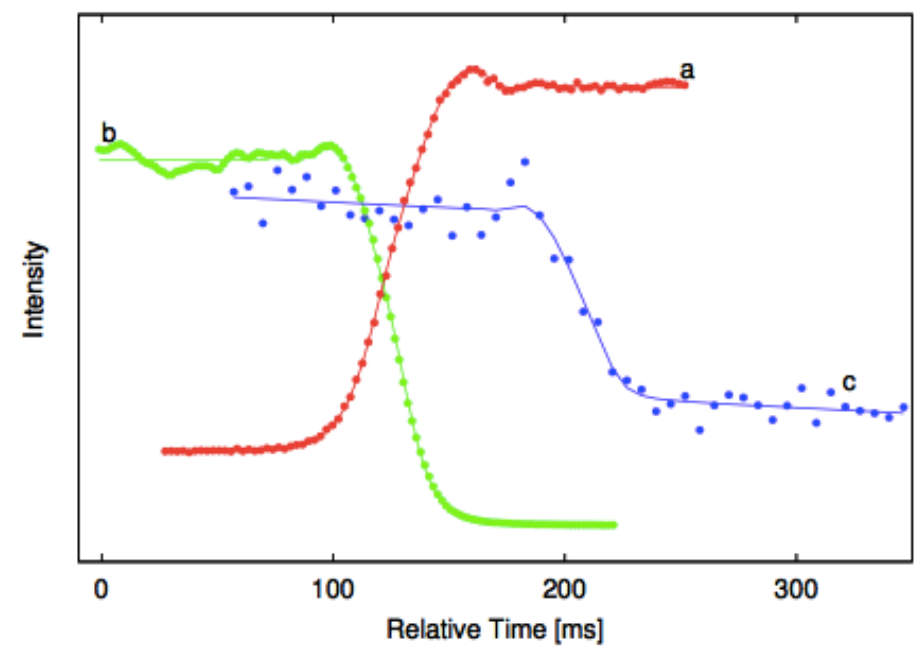

Figure 2: Light curves (points) marked as a, b, c, from the SAO 6-m (red), Devasthal 1.3-m (green), and TNT 2.4-m (blue) telescopes, respectively. The data have been shifted in time, scaled and shifted in intensity, to fit in a single figure. The solid lines are the best fit by a uniform-disc (UD) model in each case. (Taken from Richichi et al. 2017)

\subsection{Post Common-envelope Binary SDSS J1021+1744}

SDSS J1021+1744 is a detached, eclipsing white dwarf/M dwarf binary discovered in the Sloan Digital Sky Survey (SDSS). Outside the primary eclipse, the light curves of such systems are usually smooth and characterized by low-level variations caused by tidal distortion and heating of the M star component. Early data on SDSS J1021+1744 obtained in June 2012 by Parsons et al. (2013) using the Liverpool Telescope were showing an unusual dip in the flux of uncertain origin shortly after the white dwarf's eclipse. Irawati et al. (2016) presented high-time resolution, multi-wavelength observations of 35 more eclipses over 1.3 yrs, showing that the dip has a lifetime extending over many orbits. Moreover, the "dip" is, in fact, a series of dips that vary in depth, number, and position, although they are always placed in the phase interval 1.06-1.26 after the white dwarf's eclipse, near the Lagrangian point $\mathrm{L}_{5}$ in this system. Since SDSS J1021+1744 is a detached binary, it follows that the dips are caused by the transit of the white dwarf by material around $L_{5}$. A possible interpretation is that they are the signatures of prominences, a phenomenon already known from $\mathrm{H} \alpha$ observations of rapidly rotating single stars as well as binaries. Fig. 3 shows a close look of light curves of SDSS J1021+1744 between phases 1.05 and 1.26. What makes SDSS J1021+1744 peculiar is that the material is dense enough to block continuum light. The dips appear to have finally faded out around May 2015 after the first detection by Parsons et al. in 2013, suggesting a lifetime of years.

\subsection{Intra-night Variables in the Young Star Cluster Stock 8}

We have carried out time series observations of the young star cluster Stock 8 . The cluster was observed in 7 nights during January 2015 - January 2016 using ULTRASPEC mounted on the 2.4-m TNT. The central $7.7^{\prime} \times 7.7^{\prime}$ area (the FoV of ULTRASPEC) of the cluster is observed in order to identify variables in that region. The images were obtained in SDSS $g^{\prime}$ and $i^{\prime}$ filters. From our time series photometry, we identified 49 short period variable stars in the cluster (paper in preparation). Fig. 4 shows the light curves of a few pre-main sequence stars. Thirteen main-sequence and 16 premain-sequence stars are found to be probable members of the cluster. The main-sequence stars are classified as slowly pulsating B stars , $\beta$ Cephei stars, and $\delta$ Scuti stars. Six main-sequence stars may belong to new class variables as discussed by Mowlavi et al. (2013). The age and mass of identified 


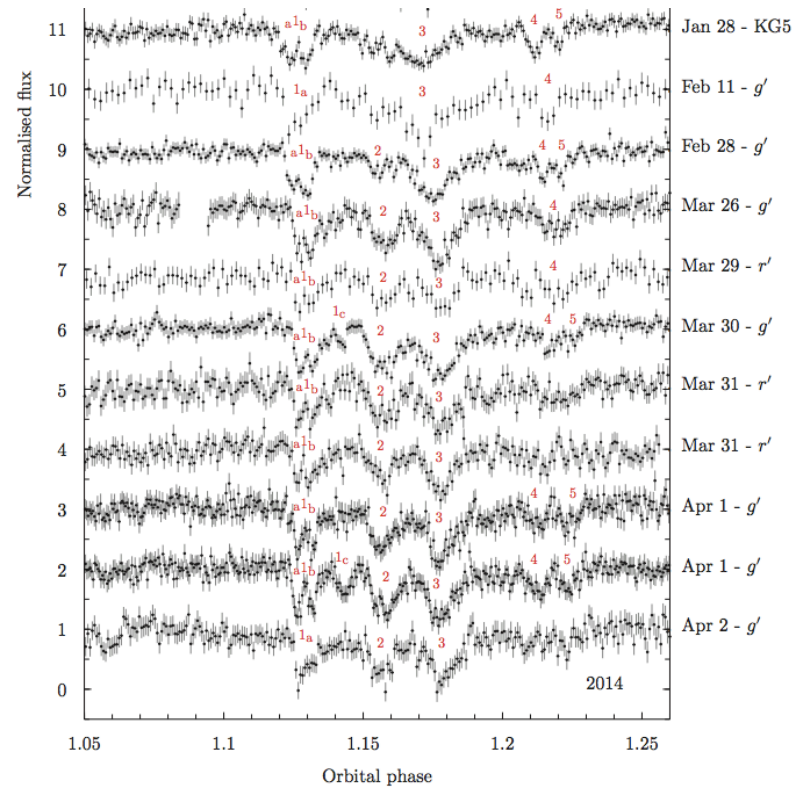

Figure 3: A close up look of the light curves of SDSS J1021+1744 between phases 1.05 and 1.26. The light curves are arranged from the oldest at the top to the newest at the bottom. The position of each dip is marked with numbers from 1 to 5 . In some light curves where dip 1 is split into three smaller dips, we annotated them with $1 \mathrm{a}, 1 \mathrm{~b}$ and $1 \mathrm{c}$. Each mark represents one dip, except for alb where we count two dips. The total number of dips for each light curve is counted based on these marks. (Taken from Irawati et al. 2016)

pre-main-sequence stars are found to be $\lesssim 5 \mathrm{Myr}$ and in the range of $0.5 \lesssim M_{\star} / \mathrm{M}_{\odot} \lesssim 2.8$, and hence these could be T Tauri stars. It is found that the amplitude of variability decreases with increase in mass, which could be due to the dispersal of the disk of relatively massive stars. The present sample of short period variables indicates that relatively massive T Tauri stars $\left(\sim 2 \mathrm{M}_{\odot}\right)$ are slow rotators in comparison to T Tauri stars having masses $\sim 0.5-1.0 \mathrm{M}_{\odot}$. Some T Tauri stars with an accreting disk are found to be fast rotators which is against expectations of disk-regulated angular momentum evolution.

\section{Proposed Three-Channel CCD Photometer at the 3.6-m DOT}

In the previous sections, we have described the capabilities of ULTRASPEC and a few recent science results obtained with that instrument. ULTRACAM, out of which the ULTRASPEC has emerged, is a three-channel fast photometer and hence can simultaneously record images in three filters. Multicolour CCD imaging can benefit the study of a variety of astrophysical objects. The main purpose of multicolour photometry is to measure the spectral energy distribution (SED) and/or its evolution for variable objects such as variable stars, active galactic nuclei, and gamma-ray burst afterglows (e.g. Greiner et al. 2008), in order to understand the underlying physics (e.g., Warner 2006).

A three-channel camera like ULTRACAM can also be useful for the recently installed modern 3.6-m Devasthal Optical Telescope (DOT). The telescope is installed at Devasthal: a new observing station of the Aryabhatta Research Institute of observational sciencES (ARIES). A $4 \mathrm{~K} \times 4 \mathrm{~K}$ CCD Imager, Faint Object Spectrograph and Camera (FOSC) and TIFR-ARIES Near Infrared Spectrometer (TANSPEC) are among the first generations instruments on the DOT. As a second generation instrument, ARIES has proposed for a three-channel fast CCD camera similar to ULTRACAM in collaboration with Vik Dhillon and his team in the UK. It should be noted that this project is still in 

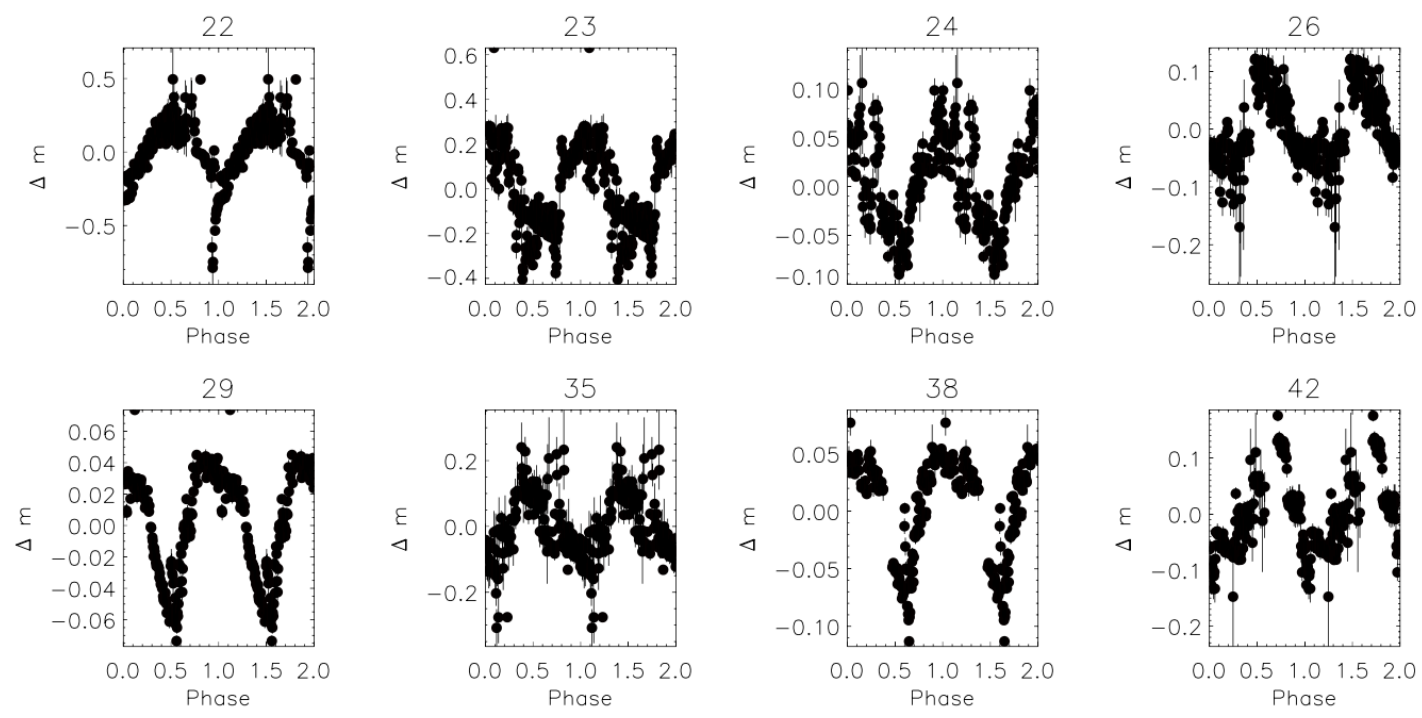

Figure 4: The g'-band phased light curves of a PMS variable stars (under preparation).

the very beginning stage and discussions between the ULTRACAM team and ARIES are on-going (private communication with Vik Dhillon). Dhillon had suggested that the proposed camera can tentatively be a three-channel (any of the $u^{\prime} g^{\prime} r^{\prime}, u^{\prime} g^{\prime} i^{\prime}$ and $u^{\prime} g^{\prime} z^{\prime}$ combinations), $13 \mu \mathrm{m} / \mathrm{pixel}, 1024 \times 1024$ EMCCD with a plate scale of $0.37^{\prime \prime} /$ pixel. In order to get a better idea of a three-channel CCD photometer, we refer to the ULTRACAM paper by Dhillon et al. (2007b).

\section{Acknowledgments}

RKY would like to thank the organizers for giving the opportunity to speak and for providing partial financial support.

\section{References}

Dhillon V. S., Marsh T. R., Stevenson M. J. et al. 2007, MNRAS, 378, 825

Dhillon V., Marsh T., Copperwheat C. et al. 2007, The Messenger, 127, 41

Dhillon V. S., Marsh T. R., Atkinson D. C. et al. 2014, MNRAS, 444, 4009

Greiner J., Bornemann W., Clemens C. et al. 2008, PASP, 120, 405

Irawati P., Richichi A., Bours M. C. P. et al. 2016, MNRAS, 456, 2446

Littlefair S. P., Dhillon V. S., Marsh T. R. et al. 2006, Science, 314, 1578

Marsh T. R., Parsons S. G., Bours M. C. P. et al. 2014, MNRAS, 437, 475

Mowlavi N., Barblan F., Saesen S. et al. 2013, A\&A, 554, 10

Ortiz J. L., Sicardy B., Braga-Ribas F. et al. 2012, Nature, 491, 566

Parsons S. G., Gansicke, B. T., Marsh T. R. et al. 2013, MNRAS, 429, 256

Richichi A., Irawati, P., Soonthornthum B. et al. 2014, AJ, 148, 100

Richichi A., Dyachenko V., Pandey A. K. et al. 2017, MNRAS, 464, 231

Simons D. A. 1995, Technical note no. 30, Gemini Observatory

Warner, B. D. 2006, A Practical Guide to Lightcurve Photometry and Analysis (1st ed.; Berlin: Springer Verlag)

Zalucha A., Fitzsimmons A., Elliot J. L. et al. 2007, Icarus, 192, 503 